Advances in Dynamical Systems and Applications.

ISSN 0973-5321, Volume 15, Number 1, (2020) pp. 115-121

(C) Research India Publications

https://dx.doi.org/10.37622/ADSA/15.1.2020.115-121

\title{
Analytic Exact Forms of Heaviside and Dirac Delta Function
}

\author{
J. Venetis \\ Section of Mechanics, Faculty of Applied Mathematics and Physical Sciences, \\ National Technical University of Athens
}

\begin{abstract}
In this paper, the author derives analytic exact forms of Heaviside and and Dirac delta function, which evidently constitute fundamental concepts of Operational Calculus and they are also involved in many other fields of applied mathematics.

In particular, these important functions are exhibited in a very simple manner by the aid of complex algebraic representations. The novelty of this work is that the proposed exact formulae are not performed in terms of non elementary special functions, e.g. Bessel functions, Error function etc and also are neither the limit of a function, nor the limit of a sequence of functions with point wise or uniform convergence.

Hence, these formulae may be much more appropriate and useful in the computational procedures which are inserted into Operational Calculus techniques along with other engineering practices.
\end{abstract}

Keywords: Heaviside function, Dirac delta function, exact form, Euler's identity

\section{INTRODUCTION}

The Heaviside step function, or unit step function, which is usually notated by the symbols $\mathrm{H}$ or $\mathrm{u}$, is a discontinuous single - valued function, the value of which is zero for negative argument and equal to unity for positive argument [1]. This special function was introduced by Oliver Heaviside, who was an important pioneer in the study of electronics and also made a remarkable contribution to the field of 
Operational Calculus [2]. A very significant property of this conceptual function is that it is capable of being represented either as a piecewise constant function or as a generalized function $[1,3]$.

The unit step function is mainly used in the calculation processes of Control Theory and signal processing in order to represent a signal which switches on at a specified time and stays switched on indefinitely. This function is also implemented together with its derivative, i.e. Dirac delta function in structural engineering in order to describe various types of structural loads, e.g. off - axis four point bending of simply supported or fully constrained beams. Hence, these significant functions are indeed very useful for the necessary calculations dealing with conceptual and embodiment design procedures from the engineering viewpoint.

In the meanwhile, there are many smooth analytic approximations to the unit step function as it can be seen in the literature $[4,5,6]$. Besides, Sullivan et al [7] obtained a linear algebraic approximation to this function by means of a linear combination of exponential functions. However, the majority of all these approaches lead to closed form representations consisting of non - elementary special functions, e.g. Logistic function, Hyperfunction, or Error function and also most of its algebraic exact forms are expressed in terms generalized integrals or infinitesimal terms, something that complicates the related computational procedures. In Ref. [8] an analytic exact form of the Unit Step Function was proposed as a sum of two inverse tangent functions. Nonetheless, according to this approach the singularity structure was left ambiguous. Unfortunately, the same problem will remain if one adopts a similar analytical approach appearing in Ref. [9] to approximate Heaviside function. Also, one may point out that a serious shortcoming of these formulae is that the involved inverse trigonometric functions do not have unique definitions.

Further, in Ref. [10] this special function this function was explicitly expressed by the aid of purely algebraic representations. The novelty of this work was that the proposed explicit formula is not performed in terms of non-elementary special functions, e.g. Error function.

Finally, another elegant approximation to Heaviside step function in the form of a summation of two logarithmic functions was carried out by Murphy in Ref. [11]. In this interesting work, the author also performs an analytic form of Dirac delta function.

In the present investigation, we introduce a single - valued function which is proved to be synonymous to the Unit Step Function. Then by differentiating this function we also obtain an analytic exact expression of Dirac delta function. Here, we elucidate that both these formulae constitute purely algebraic representations and do not contain either generalized integrals or any infinitesimal quantities. In this context, they may have good prospects towards the computational procedures that concern the applications of Heaviside function in Operational Calculus, as well as in engineering practices. 


\section{TOWARDS AN ANALYTICAL EXPRESSION OF THE UNIT STEP FUNCTION}

Let us introduce the following single valued function

$f: R \rightarrow Z$ such that

$$
f(x)=\frac{x^{1 / 2} \ln i+|x|^{1 / 2}\left(\frac{\pi}{2}+2 n \pi\right)}{x^{1 / 2}\left(\ln i+(4 n+1) \frac{\pi}{2}\right)}, \forall n \in N
$$

where $i$ denotes the imaginary unit

\section{Claim}

The function $f$ is identical to the Unit Step Function over the $\operatorname{set}(-\infty, 0) \cup(0,+\infty)$.

\section{Proof}

Let us distinguish the following two cases concerning the real variable $x$ :

i) Let $x$ be a strictly negative real variable.

Then eqn. (1) yields

$$
\begin{aligned}
& f(x)=\frac{i \cdot|x|^{1 / 2} \ln i+|x|^{1 / 2}\left(\frac{\pi}{2}+2 n \pi\right)}{i \cdot|x|^{1 / 2}\left(\ln i+(4 n+1) \frac{\pi}{2}\right)} \Leftrightarrow \\
& f(x)=\frac{i \cdot \ln i+\left(\frac{\pi}{2}+2 n \pi\right)}{i \cdot\left(\ln i+(4 n+1) \frac{\pi}{2}\right)}
\end{aligned}
$$

Here we took into account that for strictly negative real variables the following equality holds:

$i \cdot|x|^{1 / 2}=x^{1 / 2}$

On the other hand Euler's identity states that

$$
i \ln i+\frac{\pi}{2}+2 n \pi \equiv 0, \quad \forall n \in N
$$

Eqn. (2) can be combined with eqn. (4) to yield 


$$
\begin{aligned}
& f(x)=\frac{i \cdot \ln i+\frac{\pi}{2}+2 n \pi}{i \cdot\left(\ln i+(4 n+1) \frac{\pi}{2}\right)} \Rightarrow \\
& f(x)=\frac{0}{i \cdot\left(\ln i+(4 n+1) \frac{\pi}{2}\right)} \Rightarrow
\end{aligned}
$$

$f(x)=0$

Thus we proved that $f(x)$ vanishes for all strictly negative real variables.

ii) Let $x$ be a strictly positive real variable

Then eqn. (1) yields

$$
\begin{aligned}
& f(x)=\frac{x^{1 / 2} \ln i+x^{1 / 2}\left(\frac{\pi}{2}+2 n \pi\right)}{x^{1 / 2}\left(\ln i+(4 n+1) \frac{\pi}{2}\right)} \Leftrightarrow \\
& f(x)=\frac{\ln i+\frac{\pi}{2}+2 n \pi}{\ln i+(4 n+1) \frac{\pi}{2}} \Leftrightarrow \\
& f(x)=\frac{\ln i+\frac{\pi}{2}+2 n \pi}{\ln i+\frac{\pi}{2}+2 n \pi} \Leftrightarrow \\
& f(x)=1
\end{aligned}
$$

Thus we proved that $f(x)$ equals unity for all strictly positive real variables.

Consequently the function which is defined by eqn. (1) coincides with Heaviside Step Function over the $\operatorname{set}(-\infty, 0) \cup(0,+\infty)$.

Next, to obtain Dirac delta function on the basis of eqn. (1) let us set for facility reasons $n=0$

Hence, eqn. (1) yields

$$
f(x)=\frac{x^{1 / 2} \ln i+|x|^{1 / 2}\left(\frac{\pi}{2}\right)}{x^{1 / 2}\left(\ln i+\frac{\pi}{2}\right)}
$$


Then by differentiating eqn. (7) with respect to variable $x$ we infer

$$
\begin{aligned}
& \frac{d f(x)}{d x}=\frac{1}{\ln i+\frac{\pi}{2}} \cdot \frac{d}{d x}\left(\frac{x^{1 / 2} \ln i+\frac{\pi}{2}|x|^{1 / 2}}{x^{1 / 2}}\right) \Leftrightarrow \\
& \frac{d f(x)}{d x}=\frac{\frac{d}{d x}\left(x^{1 / 2} \ln i+\frac{\pi}{2}|x|^{1 / 2}\right) \cdot x^{1 / 2}-\left(x^{1 / 2} \ln i+\frac{\pi}{2}|x|^{1 / 2}\right) \frac{d}{d x}\left(x^{1 / 2}\right)}{x} \Leftrightarrow \\
& \frac{d f(x)}{d x}=\frac{x^{1 / 2} \cdot\left(\frac{\ln i}{2 x^{1 / 2}}+\frac{\pi \cdot x}{4|x|^{3 / 2}}\right)-\frac{x^{1 / 2} \ln i+\frac{\pi}{2}|x|^{1 / 2}}{2 x^{1 / 2}}}{\left(\ln i+\frac{\pi}{2}\right) \cdot x}
\end{aligned}
$$

Thus we obtained an analytic exact form of Dirac delta function over the set $(-\infty, 0) \cup(0,+\infty)$ in terms of complex quantities.

\section{DISCUSSION}

In the previous unit, we gave a rigorous proof, that all the elements of the set of single valued functions $f: R \rightarrow Z$ which are defined by eqn. (1) for any natural number $n$ coincide with the Heaviside step function over the $\operatorname{set}(-\infty, 0) \cup(0,+\infty)$. In the sequel, we derived an analytic exact form of Dirac delta function.

However, one may pinpoint that a shortcoming of the single - valued function introduced by eqn. (1) is that it cannot be determined at $x=0$. Here, one may emphasize that when Heaviside step function is approached by the logistic function it is admitted beforehand that $H(0)=\frac{1}{2}$.

In addition, one may observe that both eqn. (1) and eqn. (8) contains imaginary quantities.

Indeed according to Euler's identity, $\ln i=i \cdot\left(\frac{\pi}{2}+2 n \pi\right), \forall n \in N$.

This fact may diminish the practical use of the presented formulae concerning computational procedures, since in applied mathematics, the Dirac (delta) function is often manipulated as a kind of limit (a weak limit) of a sequence of functions, each 
member of which has a tall spike at the origin, e.g. a sequence of Gaussian distributions centered at the origin with variance tending to zero. Moreover, one should take into consideration that as integration of the delta function results in the Heaviside step function, it follows that the static deflection of a slender beam subject to multiple point loads is described by a set of piecewise polynomials.

\section{CONCLUSIONS}

The objective of this work was to propose an analytic exact form of Unit Step function along with Dirac delta function.

The proposed formulae, constitute purely algebraic representations and do not contain either generalized integrals or any infinitesimal quantities. Further they do not contain other special functions e.g. Bessel functions, Error function etc. In this famework, thay may have good prospects towards the computational procedures that concern the applications of the Unit Step and delta function in Operational Calculus, as well as in many engineering practices. However, we have to elucidate that a shortcoming of eqn.

(1) which furnishes Heaviside step function, is that it cannot be determined at $x=0$.

\section{REFERENCES}

[1] Abramowitz, M. and Stegun, I. A. (1972). Handbook of Mathematical Functions with Formulas, Graphs and Mathematical Tables, Dover Publications INC

[2] Hunt, B. J. (2012). Oliver Heaviside. Phys. Today, 65(11), 48.

[3] Bracewell, R. (2000). Heaviside's Unit Step Function,H(x). The Fourier Transform and its Applications, McGraw - Hill

[4] Berg L. (1967). Introduction to the Operational Calculus, North - Holland Publishing Company

[5] Kanwal, R. P. (1998). Generalized Functions Theory and Technique: Theory and Technique. 2nd ed. Boston, MA: Birkhäuser

[6] Spanier, J. and Oldham, K. B. (1987). The Unit-Step u(x-a) and Related Functions Ch. 8 from: An Atlas of Functions. Washington, DC: Hemisphere, 63-69.

[7] Sullivan J. Crone L. Jalickee J. (1980) Approximation of the Unit Step Function by a Linear Combination of Exponential Functions, Journal of. Approximation Theory, 28, $299-308$

[8] J. Venetis, An analytic exact form of the unit step function, Mathematics and Statistics 2(7) (2014), 235-237.

[9] J. Venetis and E. Sideridis, Study of asymmetric elastic beams in off-axis four-point bending, International Review of Aerospace Engineering 8(6) (2015), 185-197. 
[10] J. Venetis, An analytic exact form of the Heaviside step function Advances and Applications in Discrete Mathematics, Volume 22, Number 2, 2019, Pages 153-159

[11] M. Kyle Murphy, Explicit Forms of Discontinuous Functions, the Dirac Delta, and Irreducible Forms (Preprint)

https://www.academia.edu/11704122/Explicit_Forms_of_Discontinuous_Functions the Dirac_Delta and Irreducible_Forms 
